\title{
Stochastic Macromodeling of Nonlinear Systems via Polynomial Chaos Expansion and Transfer Function Trajectories
}

\author{
Domenico Spina $^{\dagger}$, Dimitri De Jonghe ${ }^{\ddagger}$, Student Member, IEEE, Dirk Deschrijver ${ }^{\dagger}$, Senior Member, IEEE, \\ Georges Gielen ${ }^{\ddagger}$, Fellow, IEEE, Luc Knockaert ${ }^{\dagger}$, Senior Member, IEEE, Tom Dhaene ${ }^{\dagger}$, Senior Member, IEEE
}

\begin{abstract}
A novel approach is presented to perform stochastic variability analysis of nonlinear systems. The versatility of the method makes it suitable for the analysis of complex nonlinear electronic systems. The proposed technique is a variation-aware extension of the Transfer Function Trajectory method by means of the Polynomial Chaos expansion. The accuracy with respect to the classical Monte-Carlo analysis is verified by means of a relevant numerical example showing a simulation speedup of $1777 X$.
\end{abstract}

Index Terms-Nonlinear systems, variability analysis, polynomial chaos, transfer function trajectories.

\section{INTRODUCTION}

The evaluation of the effects of geometrical or electrical parameter variability on the performance of modern microwave components and circuits is fundamental, due to the increasing complexity, density and bandwidth of these circuits [1], [2]. The Monte-Carlo (MC) method is the standard for the variability analysis due to its accuracy and robustness. It has however the clear limitation of requiring a large number of simulations. Indeed, the simulation of complex, high-speed microwave components and circuits can be expensive, in terms of both memory and computational time.

A reliable alternative to MC-based approaches is represented by the Polynomial Chaos (PC) expansion [3]-[8] which describes a stochastic process as a series of orthogonal polynomials with suitable coefficients. The PC expansion has extensively been applied to the variability analysis of linear systems (i.e. lumped elements circuits [9], [10], multiconductor transmission lines [11]-[13] and generic linear multiport system [14]). In particular, the contribution [14] demonstrates the accuracy and efficiency of the frequencydomain variability analysis performed while applying the PC expansion to the state-space description of the linear system under study. However, the application of the PC expansion to the variability analysis of nonlinear electronic devices so far has been limited to

- specific types of circuits (namely oscillators [15] and DC/DC converters [16]);

$\dagger$ : Department of Information Technology, Internet Based Communication Networks and Services (IBCN), Ghent University - iMINDS, Gaston Crommenlaan 8 Bus 201, B-9050 Gent, Belgium, email: \{domenico.spina, Dirk.Deschrijver, luc.knockaert, tom.dhaene\}@intec.ugent.be;

$\ddagger$ : MICAS Laboratories, ESAT, KU Leuven, B-3001 Leuven, Belgium, e-mail: gielen@esat.kuleuven.be.

Manuscript received xxxxx, 2014.
- the evaluation of the effects of variability of macromodels embedded in nonlinear circuits [17].

In this paper a novel method is proposed for the variability analysis of complex nonlinear systems based on the calculation of the PC expansion of the Transfer Function Trajectory (TFT) [18]-[20] model of the system under study, indicated below as the PC-TFT model. The proposed technique is particularly suitable to model radio frequency (RF) nonlinear circuits thanks to the modeling power of the parametric vector fitting (VF) algorithm [21]-[23] employed by the TFT method.

The calculation of the PC-TFT model requires a two-step process. At first a discrete number of TFT models is computed corresponding to a discrete set of samples of time and of geometrical or physical parameters chosen for the variability analysis. Then the desired PC-TFT model is computed using the linear regression approach [4]. Finally, the variability analysis of the system is performed accurately and efficiently by using the PC model of the system's output, which can directly be obtained from the PC-TFT model by solving a suitable system of ordinary differential equations (ODEs) using a standard numerical method.

The proposed method offers several advantages: it can be applied to a broad range of nonlinear circuits thanks to the modeling power of the TFT; it offers the efficiency and accuracy of the PC method in performing the variability analysis; and it is suitable for the analysis of complex nonlinear circuits since a hierarchical approach can be used for the calculation of the PC-TFT model.

This paper is structured as follows. First, an overview of the PC theory and of the TFT trajectory method is given in Section II and III, respectively. The time-domain stochastic macromodeling technique is described in Section V, and relevant numerical examples are presented in Section VI, validating the proposed technique. Conclusions are summed up in Section VII.

\section{Preliminaries: PC PROPERTIES}

The PC expansion describes a stochastic process in matrix form $\boldsymbol{Y} \in \mathbb{R}^{U \times V}$ with finite variance [3], [14] as a series of orthogonal polynomials $\varphi_{i}(\boldsymbol{\xi})$ depending on a vector of normalized random variables $\boldsymbol{\xi}$ with suitable coefficients $\boldsymbol{\alpha}_{i} \in$ $\mathbb{R}^{U \times V}$ as [3], [4]:

$$
\boldsymbol{Y}=\sum_{i=0}^{\infty} \boldsymbol{\alpha}_{i} \varphi_{i}(\boldsymbol{\xi})
$$


In particular, the polynomials in equation (1) are orthogonal with respect to a probability measure $W(\boldsymbol{\xi})$ with support $\Omega$ according to [5]:

$$
<\varphi_{i}(\boldsymbol{\xi}), \varphi_{j}(\boldsymbol{\xi})>=\int_{\boldsymbol{\Omega}} \varphi_{i}(\boldsymbol{\xi}) \varphi_{j}(\boldsymbol{\xi}) W(\boldsymbol{\xi}) d \boldsymbol{\xi}=a_{i} \delta_{i j}
$$

where $a_{i}$ are positive numbers and $\delta_{i j}$ is the Kronecker delta.

The infinite expansion (1) is an exact description of the stochastic process under study, but for practical implementation the series must be truncated to a limited number of $M+1$ basis functions, leading to the finite PC model:

$$
\boldsymbol{Y} \approx \sum_{i=0}^{M} \boldsymbol{\alpha}_{i} \varphi_{i}(\boldsymbol{\xi})
$$

The main advantage of the PC model is the efficient and accurate representation of the system variability. Indeed, the mean $\boldsymbol{\mu}$ and the variance $\boldsymbol{\sigma}^{2}$ of the stochastic process $\boldsymbol{Y}$ can be expressed as [4]:

$$
\begin{aligned}
\boldsymbol{\mu} & =\boldsymbol{\alpha}_{0} \\
\boldsymbol{\sigma}^{2} & =\sum_{i=1}^{M} \boldsymbol{\alpha}_{i}^{2}<\varphi_{i}(\boldsymbol{\xi}), \varphi_{i}(\boldsymbol{\xi})>
\end{aligned}
$$

Apart from all moments, also stochastic functions of $\boldsymbol{Y}$, such as the probability density function (PDF) and the cumulative density function (CDF), can be computed following standard analytical formulas or numerical schemes [6].

In the following, a methodology is presented to obtain the PC model (3) for the case of independent random variables $\boldsymbol{\xi}$. The approaches described in [4], [5], [7] can be adopted for correlated random variables.

The orthogonal polynomials in (3) (also referred to as basis functions) can be computed as product combinations of orthogonal polynomials corresponding to each individual random variable $\xi_{i}$ [4], [7]. Indeed, the global uncertainty PDF is the product of the PDFs of the single random variables, and the probability measure $W(\boldsymbol{\xi})$ becomes:

$$
W(\boldsymbol{\xi})=\prod_{i=1}^{Z} W_{i}\left(\xi_{i}\right)
$$

where $Z$ is the number of random variables. Furthermore, it can be proven that the total number of basis functions $M+1$ used in the PC model (3) is [4]:

$$
M+1=\frac{(Z+P) !}{Z ! P !}
$$

where $P$ is the highest degree of the polynomials used in the PC model (3).

Finally, the basis functions can be calculated numerically for independent random variables with arbitrary PDFs following the approach described in [5], while for random variables with specific PDFs the corresponding basis functions are the polynomials of the Wiener-Askey scheme [8]. For example, in the uniform PDF case the basis functions are the Legendre polynomials, while in the Gaussian PDF case the basis functions are the Hermite polynomials.

Upon determination of the basis functions, the corresponding PC coefficients $\boldsymbol{\alpha}_{i}$ for $i=0, \ldots, M$ can be computed following one of the two main methods described in the literature: the spectral projection and the linear regression technique [4].

For a complete reference on PC theory, the reader is referred to $[3]-[8]$.

\section{Piecewise TFT Modeling}

We consider nonlinear dynamical systems of order $N$ with a state-space description that arises when modeling electric circuits by modified nodal analysis (MNA):

$$
\dot{\boldsymbol{g}}(\boldsymbol{x}(t))=\boldsymbol{f}(\boldsymbol{x}(t))+\boldsymbol{B} \boldsymbol{u}(t), \boldsymbol{y}(t)=\boldsymbol{C}^{T} \boldsymbol{x}(t)
$$

In this paper, $\boldsymbol{x}(t) \in \mathbb{R}^{N}$ is the state vector corresponding to the node voltages and inductor currents in the circuit and $\boldsymbol{u}(t) \in \mathbb{R}^{M_{i}}$ are the inputs to the circuit. $\boldsymbol{g}(\cdot)$ and $\boldsymbol{f}(\cdot) \in$ $\mathbb{R}^{N \times N}$ are matrix-valued functions describing the charges and currents of nonlinear components. $\boldsymbol{B} \in \mathbb{R}^{N \times M_{i}}$ is a constant incidence matrix, which maps the inputs to the internal nodes of the circuit. $\boldsymbol{C} \in \mathbb{R}^{N \times M_{o}}$ is the output matrix and $\boldsymbol{y}(t) \in$ $\mathbb{R}^{M_{o}}$ denotes the output variables.

Trajectory piecewise (TPW) methods have proven themselves to be state-of-the-art in the field of accurate automated model generation [24], [25]. Here, the state space is covered with linear or low-order polynomial snapshots of the nonlinear system (8). Consequently, the nonlinear matrix functions $\boldsymbol{f}(\cdot)$ and $\boldsymbol{g}(\cdot)$ are approximated over a significant portion of the reachability space [24], [26] by a convex combination of affine functions:

$$
\begin{aligned}
& \boldsymbol{f}(\boldsymbol{x}) \approx \sum_{j} w_{j}(\boldsymbol{x})\left(\boldsymbol{A}_{j} \boldsymbol{x}+\mathbf{a}_{j}\right) \\
& \boldsymbol{g}(\boldsymbol{x}) \approx \sum_{j} w_{j}(\boldsymbol{x})\left(\boldsymbol{E}_{j} \boldsymbol{x}+\mathbf{e}_{j}\right)
\end{aligned}
$$

where $A_{j}, E_{j}$ are the Jacobians of the linearization around $\boldsymbol{x}_{j}=\boldsymbol{x}\left(t_{j}\right)$ and $\mathbf{a}_{j}, \mathbf{e}_{j}$ are inhomogeneous offset terms. The scalar function $w_{j}(\boldsymbol{x})$ performs a weighted interpolation between the samples such that $w_{j}\left(\boldsymbol{x}_{j}\right)=1$. The idea of generating a collection of local models has also been used for the design of gain-scheduled controllers and is referred to as a quasi-linear parameter-varying (quasi-LPV) representation of the nonlinear system [26]. For robustness, the samples that are included in the quasi-LPV model need to cover the reachability space of the system. In practice, it often suffices to apply typical training signals with a large amplitude over a frequency range of interest.

The linearized matrices $\boldsymbol{A}_{j}, \boldsymbol{E}_{j}$ are large and sparse, so projection-based model-order reduction techniques can be applied for reducing their complexity to an order $R \ll N$ [27], [28]. More recently, the transfer function trajectory (TFT) representation was proposed as a scalable version of the TPW approach that guarantuees global stability by transforming the linearized samples to the frequency domain [19]:

$$
\boldsymbol{H}_{j}(s)=\boldsymbol{C}^{T}\left(s \boldsymbol{E}_{j}+\boldsymbol{A}_{j}\right)^{-1} \boldsymbol{B}
$$

The above collection of transfer functions $\boldsymbol{H}_{j}(s)$ is parameterized in the frequency $s$ and the state space index $j=1, \ldots, T$, with $T$ the number of state space samples. The resulting hyperplane is then approximated along the frequency axis with $R \ll N$ fixed poles $\operatorname{diag}(\widehat{\boldsymbol{A}})$ using the parametric 
VF algorithm [22], [23]. Due to the non-uniqueness of the frequency-domain representation, the pole-residue form that is computed by the VF algorithm has multiple realizations which are related by similarity transform. For example, the Hammerstein and Wiener realizations respectively become:

$$
\begin{aligned}
& \boldsymbol{H}_{j}(s) \approx \widehat{\boldsymbol{C}}^{T}(s \boldsymbol{I}-\widehat{\boldsymbol{A}})^{-1} \widehat{\boldsymbol{B}}_{j} \quad \text { for } j=1, \ldots, T \\
& \boldsymbol{H}_{j}(s) \approx \widehat{\boldsymbol{C}}_{j}^{T}(s \boldsymbol{I}-\widehat{\boldsymbol{A}})^{-1} \widehat{\boldsymbol{B}} \quad \text { for } j=1, \ldots, T
\end{aligned}
$$

The nonlinear functionality of the system approximation is fully embedded in the residues $\widehat{\boldsymbol{r}}_{l, k}$ by fixing the poles of the model over the entire state space. Moreover, the model is assured to be bounded-input bounded-output (BIBO) stable using a simple pole-flipping scheme [22].

In the remainder of this paper, the TFT model (12) is implemented as a multi-channel Wiener system with a linear time-invariant block at the input and a nonlinear readout map. In order to capture strongly nonlinear dynamics, the nonlinear part is implemented as a piecewise combination of linear functions. Other nonlinear function approximations of the residues can be found in [18]-[20]. The time-domain representation of the Wiener-TFT approximation is found by piecewise integration of the residues:

$$
\left\{\begin{array}{l}
\dot{\hat{\boldsymbol{x}}}(t)=\widehat{\boldsymbol{A}} \widehat{\boldsymbol{x}}(t)+\widehat{\boldsymbol{B}} \boldsymbol{u}(t) \\
\widehat{\boldsymbol{y}}(t)=\sum_{j} w_{j}(\widehat{\boldsymbol{x}}(t))\left(\widehat{\boldsymbol{C}}_{j}^{T} \widehat{\boldsymbol{x}}(t)+\widehat{\boldsymbol{D}}_{j}\right)
\end{array}\right.
$$

The constant $\widehat{D}_{j}$ is found by comparing the system output with the output of the model with $\widehat{D}_{j}=0$. By introducing:

$$
\begin{aligned}
& \widehat{\boldsymbol{C}}(\widehat{\boldsymbol{x}})=\sum_{j} w_{j}(\widehat{\boldsymbol{x}}(t)) \widehat{\boldsymbol{C}}_{j}^{T} \\
& \widehat{\boldsymbol{D}}(\widehat{\boldsymbol{x}})=\sum_{j} w_{j}(\widehat{\boldsymbol{x}}(t)) \widehat{\boldsymbol{D}}_{j}
\end{aligned}
$$

the model (13) can be written compactly as:

$$
\begin{cases}\dot{\widehat{\boldsymbol{x}}}(t) & =\widehat{\boldsymbol{A}} \widehat{\boldsymbol{x}}(t)+\widehat{\boldsymbol{B}} \boldsymbol{u}(t) \\ \widehat{\boldsymbol{y}}(t) & =\widehat{\boldsymbol{C}}(\widehat{\boldsymbol{x}}) \widehat{\boldsymbol{x}}(t)+\widehat{\boldsymbol{D}}(\widehat{\boldsymbol{x}})\end{cases}
$$

In the following sections, a variation-aware extension of the TFT models is developed by means of the PC theory.

\section{Calculation of the PC-TFT Model}

The goal of the proposed contribution is to model the timedomain variability of a nonlinear system that depends on a vector of random variables $\boldsymbol{\xi}$. The PC model (3) of the TFT description (15) of the nonlinear system under study (indicated as PC-TFT) can be written as:

$$
\left\{\begin{aligned}
\dot{\widehat{\boldsymbol{x}}}(t) & =\widehat{\boldsymbol{A}} \widehat{\boldsymbol{x}}(t)+\widehat{\boldsymbol{B}} \boldsymbol{u}(t) \\
\widehat{\boldsymbol{y}}(t, \boldsymbol{\xi}) & =\sum_{i=0}^{M}\left(\widehat{\boldsymbol{C}}_{i}(\widehat{\boldsymbol{x}}) \widehat{\boldsymbol{x}}(t)+\widehat{\boldsymbol{D}}_{i}(\widehat{\boldsymbol{x}})\right) \varphi_{i}(\boldsymbol{\xi})
\end{aligned}\right.
$$

In a first step, the TFT model (15) of the system under study is computed over a discrete set of values of the random variables $\boldsymbol{\xi}$ and of the state space $\boldsymbol{x}(t)$, indicated as $\left[\boldsymbol{\xi}_{r}\right]_{r=1}^{K}$ and $\left[\boldsymbol{x}_{j}\right]_{j=1}^{T}$, respectively. The sampling in the state space is dictated by the training stimuli of the TFT model and does not need to be uniform [19], [24], while the samples of the random variables $\boldsymbol{\xi}$ are chosen over a regular grid in the stochastic space $\Omega$. In particular, the number $K$ of samples in the stochastic space is chosen according to:

$$
K \approx 2(M+1)=2 \frac{(Z+P) !}{Z ! P !}
$$

The TFT samples (12) that correspond to each value of $\left[\boldsymbol{\xi}_{r}\right]_{r=1}^{K}$ and $\left[\boldsymbol{x}_{j}\right]_{j=1}^{T}$ can be computed as in Section III. However, it is important to choose the same set of poles for all the TFT samples (12) computed for all the values of $\left[\boldsymbol{\xi}_{r}\right]_{r=1}^{K}$ and $\left[\boldsymbol{x}_{j}\right]_{j=1}^{T}$. Hence, the matrices $\widehat{\boldsymbol{A}}$ and $\widehat{\boldsymbol{B}}$ of all the $K \times T$ TFT samples calculated are assumed to be constant, since they are independent from both the time and the random variables considered. Furthermore, the stability of the TFT samples computed is guaranteed by enforcing the stability of the chosen set of poles [22]. Therefore, we have obtained a TFT model that is parameterized in the stochastic space and the state space:

$$
\left\{\begin{aligned}
\dot{\hat{\boldsymbol{x}}}(t) & =\widehat{\boldsymbol{A}} \widehat{\boldsymbol{x}}(t)+\widehat{\boldsymbol{B}} \boldsymbol{u}(t) \\
\widehat{\boldsymbol{y}}(t, \boldsymbol{\xi}) & =\widehat{\boldsymbol{C}}\left(\widehat{\boldsymbol{x}}_{j}, \boldsymbol{\xi}_{r}\right) \widehat{\boldsymbol{x}}(t)+\widehat{\boldsymbol{D}}\left(\widehat{\boldsymbol{x}}_{j}, \boldsymbol{\xi}_{r}\right)
\end{aligned}\right.
$$

for $r=1, \ldots, K$ and $j=1, \ldots, T$.

Finally, the desired PC-TFT model (16) can be obtained by computing the corresponding PC models of the matrices $\widehat{C}$ and $\widehat{D}$. Once the basis functions are known, as described in Section II, the PC coefficients of the matrices $\widehat{C}$ and $\widehat{D}$ can be found following the linear regression approach [4], [14], which leads to:

$$
\begin{aligned}
\boldsymbol{\Psi}_{C}\left(\boldsymbol{\xi}_{r}\right) \boldsymbol{\alpha}_{C}\left(\widehat{\boldsymbol{x}}_{j}\right) & =\boldsymbol{R}_{C}\left(\widehat{\boldsymbol{x}}_{j}, \boldsymbol{\xi}_{r}\right) \\
\boldsymbol{\Psi}_{D}\left(\boldsymbol{\xi}_{r}\right) \boldsymbol{\alpha}_{D}\left(\widehat{\boldsymbol{x}}_{j}\right) & =\boldsymbol{R}_{D}\left(\widehat{\boldsymbol{x}}_{j}, \boldsymbol{\xi}_{r}\right)
\end{aligned}
$$

where the $r$-th row of the matrices $\boldsymbol{\Psi}_{C}\left(\boldsymbol{\xi}_{r}\right)$ and $\boldsymbol{\Psi}_{D}\left(\boldsymbol{\xi}_{r}\right)$ contains the multivariate polynomial basis functions $\varphi_{i}$ for $i=0, \ldots, M$ evaluated in $\boldsymbol{\xi}_{r}$ for $r=1, \ldots, K$ multiplied by the identity matrix of the same dimension as the matrix $\widehat{C}$ and $\widehat{D}$, respectively. The corresponding set of values of the matrix $\widehat{\boldsymbol{C}}\left(\widehat{\boldsymbol{x}}_{j}, \boldsymbol{\xi}_{r}\right)$ and $\widehat{\boldsymbol{D}}\left(\widehat{\boldsymbol{x}}_{j}, \boldsymbol{\xi}_{r}\right)$ for $r=1, \ldots, K$ and $j=1, \ldots, T$ are collected in the matrix $\boldsymbol{R}_{C}\left(\widehat{\boldsymbol{x}}_{j}, \boldsymbol{\xi}_{r}\right)$ and $\boldsymbol{R}_{D}\left(\widehat{\boldsymbol{x}}_{j}, \boldsymbol{\xi}_{r}\right)$, respectively. Finally, $\boldsymbol{\alpha}_{C}\left(\widehat{\boldsymbol{x}}_{j}\right)$ contains the desired PC coefficients $\widehat{\boldsymbol{C}}_{i}\left(\widehat{\boldsymbol{x}}_{j}\right)$ and $\boldsymbol{\alpha}_{D}\left(\widehat{\boldsymbol{x}}_{j}\right)$ the PC coefficients $\widehat{\boldsymbol{D}}_{i}\left(\widehat{\boldsymbol{x}}_{j}\right)$ for $i=0, \ldots, M$ and $j=1, \ldots, T$. Note that, the realization technique used to convert a pole-residue model to a state-space form has an influence on the smoothness of the matrices in (19), (20) with respect to the design parameters and, therefore, on the accuracy of the final PC model [14]. We use a Wiener realization in our approach.

It is important to notice that the stability of the computed PC-TFT model (16) is guaranteed if a stable set of poles is used to obtain the parameterized TFT model (18).

Finally, the proposed method is efficient if the number of random variables $Z$ is limited, see (17), and if the number of state samples $T$ is not too high, since the total number of TFT samples to be calculated is $K \times T$. For example, considering $Z=15$ random variables with seventh-order polynomial chaos expansion $(P=7)$ the total number of TFT models that must be calculated for each time sample is 341088 , according to (17).

The entire flowchart of the proposed PC-TFT modeling strategy is summarized in Fig. 1. 


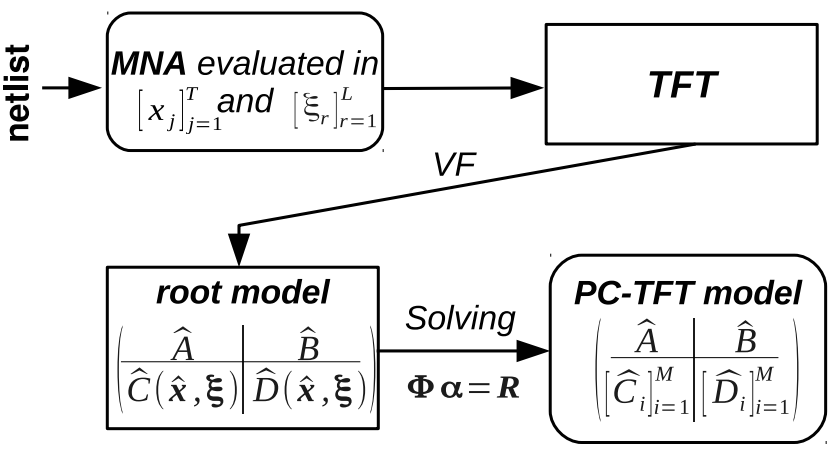

Fig. 1. Flowchart of the proposed PC-TFT modeling strategy.

\section{VARIAbiLITy ANALYSis of NONLINEAR Systems THROUGH PC-TFT MODELING}

In the following, the efficiency and accuracy of computing the PC model of the system output, starting from the corresponding PC-TFT model (16), will be demonstrated. In particular, only the PC coefficients of the system output $\widehat{\boldsymbol{y}}_{i}$ for $i=0, \ldots, M$ must be calculated. Indeed, equation (16) can be written as a system of $M+1$ independent equations, thanks to the orthogonality relation (2). Projecting (16) on the basis function $\varphi_{p}(\boldsymbol{\xi})$, for $p=1, \ldots, M$, leads to:

$$
\widehat{\boldsymbol{y}}_{p}(t)=\widehat{\boldsymbol{C}}_{p}(\widehat{\boldsymbol{x}}) \widehat{\boldsymbol{x}}(t)+\widehat{\boldsymbol{D}}_{p}(\widehat{\boldsymbol{x}})
$$

Hence, the PC coefficients of the system output can be calculated directly from the corresponding PC-TFT model by solving the following $M+1$ independent systems of ODEs:

$$
\left\{\begin{aligned}
\dot{\widehat{\boldsymbol{x}}}(t) & =\widehat{\boldsymbol{A}} \widehat{\boldsymbol{x}}(t)+\widehat{\boldsymbol{B}} \boldsymbol{u}(t) \\
\widehat{\boldsymbol{y}}_{i}(t) & =\widehat{\boldsymbol{C}}_{i}(\widehat{\boldsymbol{x}}) \widehat{\boldsymbol{x}}(t)+\widehat{\boldsymbol{D}}_{i}(\widehat{\boldsymbol{x}})
\end{aligned} \quad \text { for } i=1, \ldots, M ;\right.
$$

using a standard numerical method (e.g. Backward Euler). Note that the computational cost of solving the system of ODEs (22) can drastically be reduced by exploiting the parallelism of these calculations. Finally, to further reduce the computational cost of solving equation system (22), any numerical method that employs a non-uniform sampling in the time domain can be used in such a way as to minimize the total number $T^{\prime}$ of time samples needed, while keeping the overall accuracy. Note that the set of time samples $\left[t_{k}\right]_{k=1}^{T^{\prime}}$ depends only on numerical method chosen to solve the system of equations (22) and is independent from the particular sampling of the state space used in Section IV to build the PC-TFT model.

At this point, a PC model of the system output can be calculated over a discrete set of time samples as:

$$
\widehat{\boldsymbol{y}}\left(t_{k}, \boldsymbol{\xi}\right)=\sum_{i=0}^{M} \widehat{\boldsymbol{y}}_{i}\left(t_{k}\right) \varphi_{i}(\boldsymbol{\xi}) \quad \text { for } k=1, \ldots, T^{\prime} ;
$$

The value of the PC model (23) can easily be computed for any time sample $t_{q} \neq t_{k}$ for $k=1, \ldots, T^{\prime}$, with $t_{0}<t_{k}<t_{T^{\prime}}$ using a numerical interpolation technique [29], [30].

The proposed methodology has several advantages. Thanks to the modeling power of the TFT method, it can be applied to study a broad range of nonlinear systems, including strongly

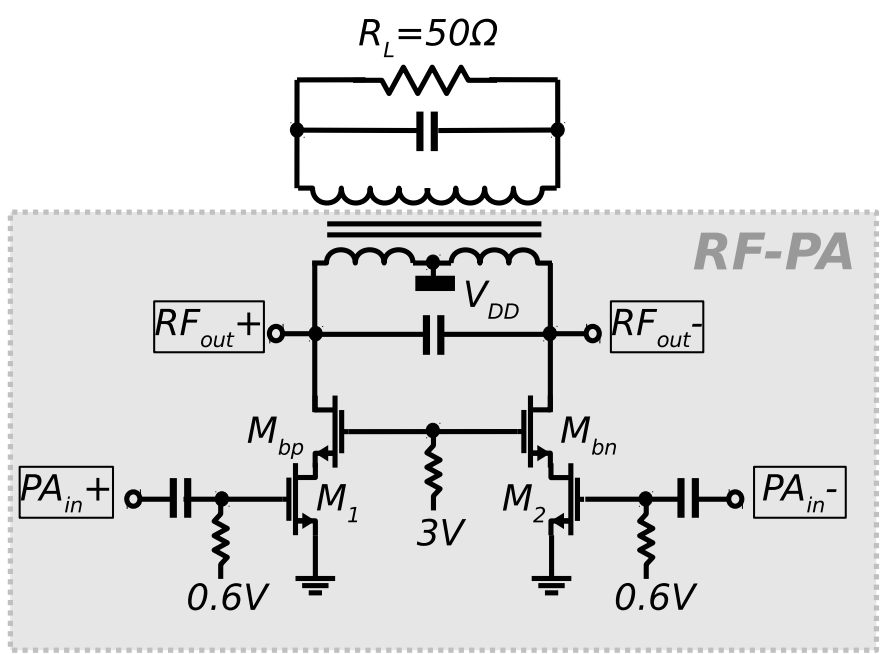

Fig. 2. Schematic representation of the RF PA circuit.

nonlinear ones, and it offers the efficiency and accuracy of the PC expansion to determine the time-domain system variability. For example, stochastic moments like (4), (5) can be determined analytically. Furthermore, the stability of the calculated PC-TFT model can be guaranteed (see Section IV). Finally, the proposed technique offers a good flexibility in modeling complex nonlinear systems. Indeed, a hierarchical approach can be used: complex nonlinear systems can be divided in simpler blocks that can be modeled separately with the proposed technique (see Section VI).

\section{NUMERICAL EXAMPLES}

The proposed PC-TFT model is demonstrated by modeling a radio frequency (RF) power amplifier (PA) circuit, see Fig. 2. The topology of the circuit was proposed in [31] and has been implemented as a netlist description in the I3T50 technology for purpose of validation of the PC-TFT technique. The circuit was stimulated by a $2 \mathrm{GHz}$ RF carrier frequency.

For this PA circuit, the analog input signals are the deterministic inputs to the model. The stochastic variables are selected based upon sensitivity screening of each stochastic variable occurring in the PA towards the output performance. This procedure yields two dominant stochastic variables $(Z=2)$ that need to be included in the model for this technology, namely the variation of the threshold voltages of the input transistors. It is assumed that the stochastic variables have a normal distribution, which is verified experimentally.

A PC model of the output mapping defined by $\widehat{C}(\cdot), \widehat{D}(\cdot)$ is computed using a fifth-order polynomial chaos expansion ( $P=5$ for accuracy reasons). Hence, the corresponding number of basis functions in the PC-TFT model is $M+1=21$, according to (7), which leads to a minimum number of samples in the stochastic space equal to $K \approx 2(M+1)=42$, according to (17). A Wiener PC-TFT model was computed using a $7-$ by -7 regular grid of the two stochastic variables $\xi$ in a range between $\pm 4 \sigma$, so $K=49$ SPICE simulations are required for each of the reference voltages. All the $K$ initial SPICE simulations are performed for 1000 time samples. From the MNA data, 49 piecewise TFT models are computed. The 


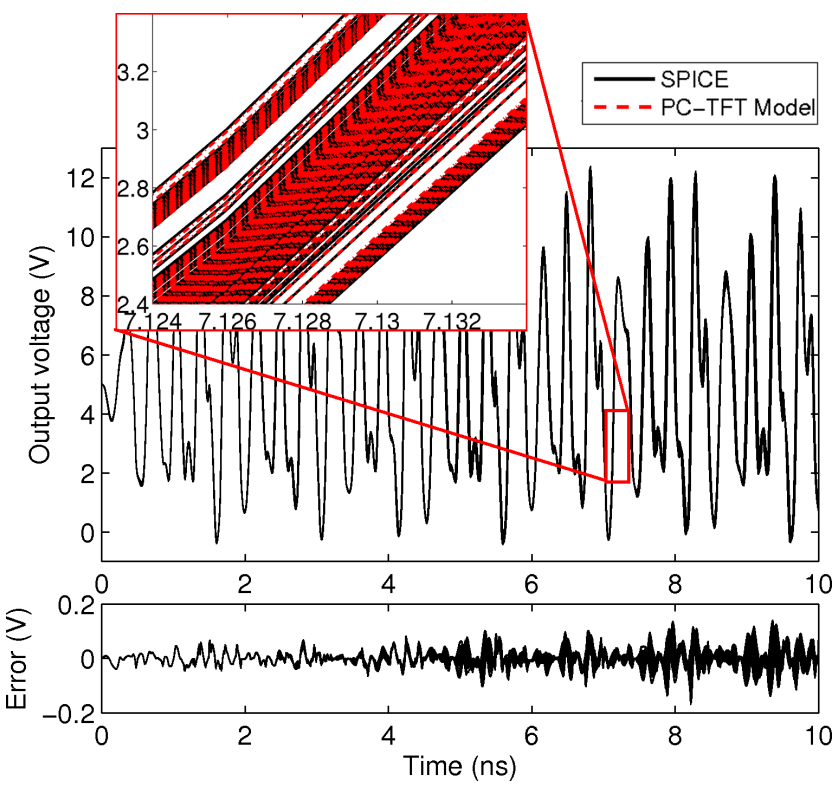

Fig. 3. Top: Time-domain waveforms of the PC circuit modeled with SPICE and the TFT model for 500 MC samples. Bottom: the difference or error between the PC-TFT model and the SPICE simulation.

computation of the 49 TFT models took $778 s$ including the training simulations. The computation of the PC model of the output mapping $\widehat{C}_{i}(\cdot), \widehat{D}_{i}(\cdot)$ in (22) took $12 s$ and the PC model (23) of the PA output $\widehat{y}_{i}$ took $1.7 \mathrm{~s}$.

The time-domain response of the PA in SPICE and of the PC-TFT model are given in Fig. 3 for 40001 transient simulations with MC sampling of the process variations that are included in the technology data of the foundry. The models were then simulated in Matlab and compared with circuitlevel SPICE. All calculations were performed on a $4 \mathrm{GHz}$ dual quad-core CPU with 12 GB RAM. It can be seen that, despite the output signal is highly dynamic and shows a strong nonlinear behavior, the model and the original circuit are almost indistinguishable. For the sake of clarity, the difference between both sets of waveforms is also plotted. The maximum difference never exceeds $0.2 \mathrm{~V}$. The evaluation of the 40001 MC samples took $4088 s$ in SPICE and only $2.3 s$ using the PC-TFT technique. Hence, a huge simulation speedup of $1777 X$ was achieved. Note that, the SPICE simulations for the $\mathrm{MC}$ analysis are performed using an adaptative timestep, for accuracy reasons. Hence, a post-processing step is necessary to be able to perform the variability analysis via MC method, since the value and the number of the time samples used for each MC run can be different with respect to the others. Indeed, the values of the output signal obtained via MC analysis are interpolated over the 1000 time samples used to compute the PC-TFT model. In order to present a fair comparison, the additional cost of the post-processing interpolation phase is not included in the computational time of the MC analysis.

More importantly, the PC-TFT model provides an analytical expression to compute stochastic moments, such as the mean $\mu$ and the variance $\sigma^{2}$ (see equations (4) and (5)) of the output waveforms. Hence, the mean and the standard deviation of the

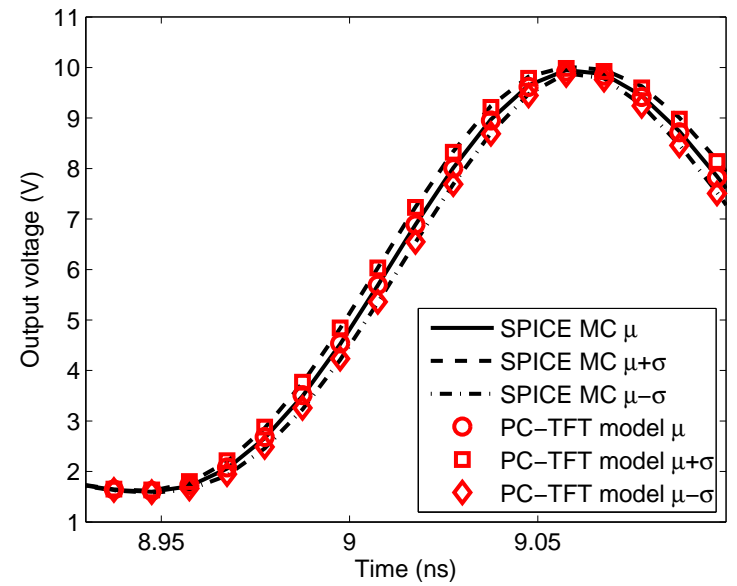

Fig. 4. The mean $\mu$ and the range $\mu \pm \sigma$ of the output voltage of the PA circuit. The black lines are computed using 40001 Monte-Carlo simulations in SPICE. The red lines are computed analytically from the PC-TFT model.

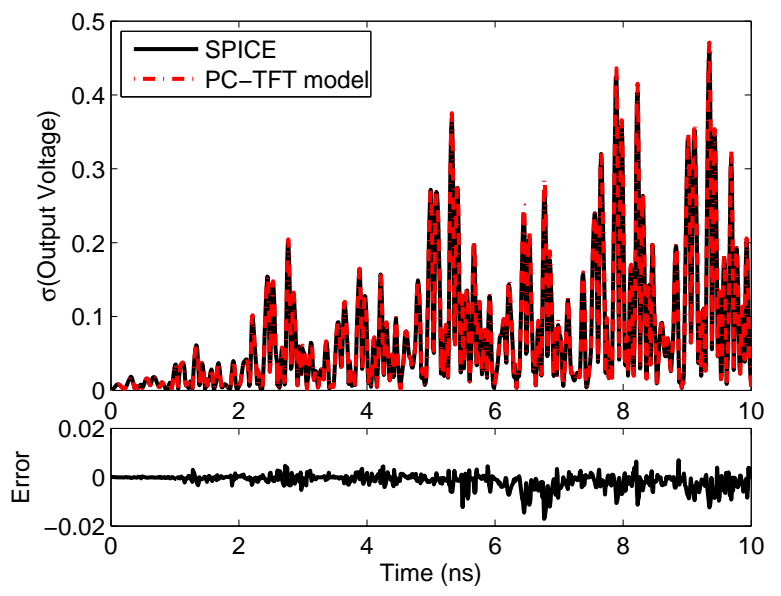

Fig. 5. Top: Standard deviation $\sigma$ of the output voltage of the PA circuit over the entire time range. The black lines are computed using 40001 MonteCarlo simulations in SPICE. The red lines are computed analytically from the PC-TFT model. Bottom: the difference or error between the PC-TFT model and the SPICE simulation.

waveform at each time point can be calculated analytically with the PC-TFT model in $0.06 s$.

The effectiveness of this approach is illustrated by comparing the mean $\mu$ and the range $\mu \pm \sigma$ of the output of the PA using both the 40001 Monte-Carlo simulations in SPICE and using the corresponding analytical expressions of the PCTFT model in Fig. 4, which has been zoomed in for the sake of visualization. Next, Fig. 5 shows the comparison of the standard deviation computed using the 40001 Monte-Carlo simulations in SPICE and using the corresponding analytical expressions of the PC-TFT model. Looking at Figs. 4, 5 it is clear that the analytical expression is very accurate without the need for a large set of Monte-Carlo samples; only $K=49$ grid samples are required for computing the PC-TFT model.

An important parameter to measure the performances of the PA considered is the total harmonic distortion (THD), defined as the ratio of the RMS amplitude of the higher harmonics and 


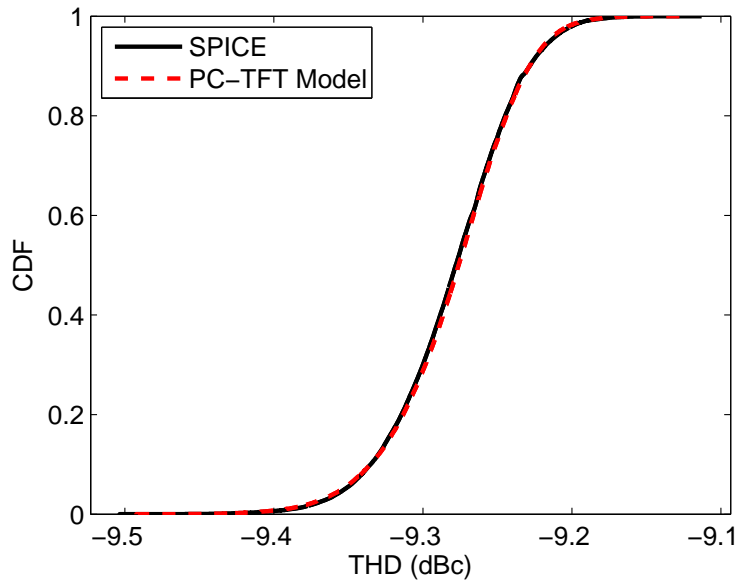

Fig. 6. Cumulative density function (CDF) of the total harmonic distortion (THD) for 40001 Monte-Carlo samples evaluated with a full SPICE simulation (black) and with the proposed PC-TFT model (red dashed line).

the fundamental harmonic when applying a $2 \mathrm{GHz}$ sinusoidal input signal.

The cumulative probability plot of the THD of the system is given in Fig. 6 for the original SPICE simulation and the PC-TFT model for 40001 MC samples.

\section{CONCLUSIONS}

This paper has presented a novel technique for the efficient variability analysis of nonlinear systems, such as amplifiers, comparators, digital filters. It is based on the use of the polynomial chaos expansion applied to the TFT description of the system under study. The proposed approach can be applied to study a broad range of nonlinear systems and a hierarchical approach can be used to reduce the modeling complexity. Finally, the method allows to perform the variability analysis with good accuracy and improved efficiency compared to Monte-Carlo analysis. Comparisons with the standard MC approach have been performed for two numerical examples, validating the accuracy and efficiency (i.e. a speedup of $1777 X$ ) of the proposed method.

\section{ACKNOWLEDGMENT}

The authors acknowledge the financial support of FP7 and ON Semiconductor Belgium BVBA in the frame of the SMAC projects and of the Interuniversity Attraction Poles Programme BESTCOM initiated by the Belgian Science Policy Office.

\section{REFERENCES}

[1] Y. Liu and J.-S. Yuan, "CMOS RF power amplifier variability and reliability resilient biasing design and analysis," IEEE Trans. Electron Devices, vol. 58, no. 2, pp. 540-546, Feb. 2011.

[2] J. S. Ochoa and A. C. Cangellaris, "Random-space dimensionality reduction for expedient yield estimation of passive microwave structures," IEEE Trans. Microw. Theory Tech., vol. 61, no. 12, pp. 4313-4321, Dec. 2013.

[3] G. Blatman and B. Sudret, "An adaptive algorithm to build up sparse polynomial chaos expansions for stochastic finite element analysis," Probabilistic Engineering Mechanics, vol. 25, no. 2, pp. 183 - 197, Apr. 2010.
[4] M. S. Eldred, "Recent advance in non-intrusive polynomial-chaos and stochastic collocation methods for uncertainty analysis and design," in Proc. 50th AIAA/ASME/ASCE/AHS/ASC Structures, Structural Dynamics, and Materials Conference, Palm Springs, California, May 2009.

[5] J. A. S. Witteveen and H. Bijl, "Modeling Arbitrary Uncertainties Using Gram-Schmidt Polynomial Chaos," in Proc. 44th AIAA Aerospace Sciences Meeting and Exhibit, no. AIAA-2006-896, Reno, Nevada, Jan. 2006.

[6] A. Papoulis, Probability, Random Variables and Stochastic Processes. McGraw-Hill College, 1991.

[7] C. Soize and R. Ghanem, "Physical systems with random uncertainties: Chaos representations with arbitrary probability measure," SIAM J. SCI. COMPUT., vol. 26, no. 2, pp. 395-410, Jul. 2004.

[8] D. Xiu and G. E. Karniadakis, "The Wiener-Askey polynomial chaos for stochastic differential equations," SIAM J. Sci. Comput., vol. 24, no. 2, pp. 619-644, Apr. 2002.

[9] Q. Su and K. Strunz, "Stochastic polynomial-chaos-based average modeling of power electronic systems," IEEE Trans. Power Electron., vol. 26, no. 4, pp. $1167-1171$, Apr. 2011.

[10] K. Strunz, Q. Su, "Stochastic formulation of SPICE-type electronic circuit simulation using polynomial chaos," ACM Trans. Model. Comput. Simul., vol. 18, no. 4, pp. 501-507, Sep. 2008.

[11] I. S. Stievano, P. Manfredi, F. G. Canavero, "Parameters variability effects on multiconductor interconnects via Hermite polynomial chaos," IEEE Trans. Compon., Packag., Manuf. Technol., vol. 1, no. 8, pp. 12341239, Aug. 2011

[12] _ "Stochastic analysis of multiconductor cables and interconnects," IEEE Trans. Electromagn. Compat., vol. 53, no. 2, pp. 501-507, May 2011.

[13] D. Vande Ginste, D. De Zutter, D. Deschrijver, T. Dhaene, P. Manfredi, F. G. Canavero, "Stochastic modeling based variability analysis of onchip interconnects," IEEE Trans. Compon., Packag., Manuf. Technol., vol. 2, no. 7, pp. $1182-1192$, Jul. 2012.

[14] D. Spina, F. Ferranti, T. Dhaene, L. Knockaert, G. Antonini, and D. Vande Ginste, "Variability analysis of multiport systems via polynomial-chaos expansion," IEEE Trans. Microw. Theory Tech., vol. 60, no. 8, pp. $2329-2338$, Aug. 2012.

[15] D. Lucor, C.-H. Su, and G. E. Karniadakis, "Generalized polynomial chaos and random oscillators," Int. J. Numer. Meth. Engng, vol. 60, no. 3, pp. 571-596, May 2004.

[16] A. Monti, F. Ponci, and T. Lovett, "A polynomial chaos theory approach to the control design of a power converter," in Proc. 35th Annual IEEE Power Electronics Specialists Conference (PESC), vol. 6, 20-25 Jun. 2004.

[17] M. R. Rufuie, E. Gad, M. Nakhla, R. Achar, "Generalized hermite polynomial chaos for variability analysis of macromodels embedded in nonlinear circuits," IEEE Trans. Compon., Packag., Manuf. Technol., vol. 4, no. 4, pp. 673-684, Apr. 2014

[18] D. De Jonghe, G. Gielen, "Efficient analytical macromodeling of large analog circuits by transfer function trajectories," in Proc. IEEE/ACM International Conference on Computer-Aided Design (ICCAD), 7-10 Nov. 2011, pp. 91-94.

[19] — "Characterization of analog circuits using transfer function trajectories," IEEE Trans. Circuits Syst. I: Regular Papers, vol. 59, no. 8, pp. 1796-1804, Aug. 2012.

[20] D. De Jonghe, D. Deschrijver, T. Dhaene, G. Gielen, "Extracting analytical nonlinear models from analog circuits by recursive vector fitting of transfer function trajectories," in Proc. Europe Conference \& Exhibition in Design, Automation \& Test (DATE), 18-22 Mar. 2013, pp. 1448-1453.

[21] B. Gustavsen and A. Semlyen, "Rational approximation of frequency domain responses by vector fitting," IEEE Trans. Power Del., vol. 14, no. 3, pp. 1052-1061, Jul. 1999.

[22] D. Deschrijver, M. Mrozowski, T. Dhaene, and D. De Zutter, "Macromodeling of multiport systems using a fast implementation of the vector fitting method," IEEE Microw. Wireless Compon. Lett., vol. 18, no. 6 , pp. 383-385, Jun. 2008.

[23] T. Dhaene, D. Deschrijver, "Stable parametric macromodeling using a recursive implementation of the vector fitting algorithm," IEEE Microw. Wireless Compon. Lett., vol. 19, no. 2, pp. 59-61, Feb. 2009.

[24] M. J. Rewieński, "A trajectory piecewise-linear approach to modelorder reduction of nonlinear dynamical systems," Ph.D. dissertation, Massachusetts Institute of Technology, 2003.

[25] R. A. Rutenbar, G. G. E. Gielen, J. Roychowdhury, "Hierarchical modeling, optimization, and synthesis for system-level analog and $\mathrm{rf}$ designs," Proceedings of the IEEE, vol. 95, no. 3, pp. 640 -669, Mar. 2007. 
[26] R. Tóth, Modeling and identification of linear parameter-varying systems. Springer, 2010.

[27] A. Odabasioglu, M. Celik, L. T. Pileggi, "PRIMA: passive reducedorder interconnect macromodeling algorithm," IEEE Trans. Comput.Aided Design Integr. Circuits Syst., vol. 17, no. 8, pp. 645-654, Aug. 1998.

[28] J. R. Phillips, L. M. Silveira, "Poor man's TBR: a simple model reduction scheme," IEEE Trans. Comput.-Aided Design Integr. Circuits Syst., vol. 24, no. 1, pp. 43-55, Jan. 2005.

[29] C. de Boor, A Practical Guide to Splines. Springer-Verlag, 1978.

[30] F. N. Fritsch and R. E. Carlson, "Monotone piecewise cubic interpolation," SIAM J. Numer. Anal., vol. 17, no. 2, pp. 238-246, Nov. 1980.

[31] B. Francois, P. Reynaert, "A fully integrated watt-level linear 900-MHz CMOS RF power amplifier for LTE-applications," IEEE Trans. Microw. Theory Tech., vol. 60, no. 6, pp. 1878-1885, Jun. 2012. 